\title{
Mucus barrier correction as a target in complex treatment of gastroesophageal reflux disease
}

\begin{abstract}
The aim of our study was to evaluate the clinical efficacy of esophageal mucus barrier correction by complex treatment with sodium hyaluronate and sodium alginate (Hyalera/ Gastropiù Bustine) application in patients with gastroesophagic reflux disease (GERD). We conducted a multicenter, randomized, open, comparative study with parallel monitoring to evaluate the effectiveness and safety of combination therapy for patients with GERD using the sodium hyaluronate and sodium alginate (Hyalera/Gastropiù Bustine). Under our supervision, there were 72 patients with GERD aged 18 to 65 years. Women were 30 $(41.7 \%)$, men $42(58.3 \%)$. The criterion for inclusion in the study was the presence of GERD symptoms (primarily heartburn) with endoscopic and morphological confirmation. Omeprazole at a dose of $20 \mathrm{mg} 2$ times a day and domperidone at a dose of $10 \mathrm{mg} 3$ times a day were used as basic therapy in both groups. In the first group, an additional sodium hyaluronate and sodium alginate (Hyalera/Gastropiù Bustine) emulsion of $15 \mathrm{ml}$ sticks (Alpiflor, Italy) was prescribed. The emulsion was administered 1 stick after a meal 3 times a day.

The frequency of heartburn in the first group after treatment was almost three times lower than in the comparison group $(\mathrm{p}=0.035)$ and they did not have nightly heartburn. Also, dysphagia was absent in the first group (almost $7 \%$ of patients remained in the second), at the same time, the incidence of belching after treatment was identical in both groups (20 and 17\%). Endoscopically, in the second group, 4.5 times more often than in the first, esophagitis was detected, and in 1 patient it was erosive. Morphologically, in patients of the first group, significant positive dynamics was noted.
\end{abstract}

Keywords: GERD, gastroesophagic reflux disease, esophagoscopy, anti-inflammatory drugs, claudins, occludins, antisecretory therapy, ECG, alcoholism, drug addiction, varying degrees, papillomatosis cells, manifestations
Volume I2 Issue I - 202I

\author{
Dorofeyev AE,' Dyadyk OO, ${ }^{2}$ Zvyagintseva \\ TD, ${ }^{3}$ Yu V Chychula, ${ }^{4}$ N.N.Rudenko, ${ }^{4}$ Stukalo \\ $\mathrm{AA}^{5}$ \\ 'Department of Internal Medicine, National Medical Academy of \\ Postgraduate Education named after P.L. Shupik, Ukraine \\ 2Department of Pathological and Topographic Anatomy, National \\ Medical Academy of Postgraduate Education named after P.L. \\ Shupik, Ukraine \\ ${ }^{3}$ Department of Gastroenterology, Kharkiv Medical Academy of \\ Postgraduate Education, Ukraine \\ ${ }^{4}$ Department of Internal Medicine, Bogomolets National Medical \\ University, Ukraine \\ ${ }^{5}$ Department of Endoscopy, Ukrainian-German \\ gastroenterological center "BIK-KIEV, Ukraine
}

\begin{abstract}
Correspondence: Dorofeyev AE, Department of Internal Medicine, National Medical Academy of Postgraduate Education named after P.L. Shupik, Ukraine, Tel 380-997693945,
\end{abstract} Email dorofeyevan@gmail.com

Received: January 18, 2021 | Published: Febrauary 12, 2021
Abbreviations: GERD, gastroesophagic reflux disease; TJ, tight junctions; PPIs, proton pump inhibitors; NMAPE, national medical academy of postgraduate education; IHA, immunohistochemical assay

\section{Introduction}

Thus, mucus barrier correction in patients with GERD could be a perspective target of the treatment. In GERD patients the addition of a proton pump inhibitor, prokinetic, sodium hyaluronate and sodium alginate (Hyalera/Gastropiù Bustine) to standard therapy can significantly (compared with the control group) reduce the frequency and severity of heartburn, the degree of damage to the esophagus according to esophagoscopy, eliminate dysphagia and nocturnal heartburn. At the same time, modification of esophageal mucus barrier correlated with clinical improvement. Morphological changes in the esophageal mucosa, with decrease in the degree of inflammation and degeneration of the epithelium, an increase in the synthesis of protective mucus and the integration of the epithelium due to claudine 1 in tight junctions induce a clinical symptoms normalisation.

According to recent epidemiological studies, a quarter of the world's population experiences symptoms of gastroesophageal reflux disease (GERD), among which the most common is heartburn. ${ }^{1}$ In Europe, the prevalence of this disease is irregular with a maximum in Greece (more than $51 \%$ of population) and a minimum in France (less than $10 \%))^{2}$ There is a $50 \%$ increase in the number of such patients compared to $1990 \mathrm{~s} .{ }^{1}$ Our country is not an exception, e.g., the incidence of GERD in Ukraine is 4.5-5.4 cases per 1000 people per year and its symptoms are observed in $15-21 \%$ of the population. ${ }^{3}$ Risk factors of GERD are well known: age over 50years, smoking, overweight/obesity, intake of non-steroidal anti-inflammatory drugs and aspirin. A frequent combination of GERD with other diseases of the gastrointestinal tract and first of all with functional dyspepsia is also reported. ${ }^{1,2}$ An important component in the pathogenesis of GERD is a decrease in the tone of the lower esophageal sphincter with the reflux of acidic gastric contents into the esophagus. However, the ability of the esophagus mucosa to resist aggressive reflux and the quality of clearance (purification) of the esophagus wall from the acid refluxed into the stomach are no less important. ${ }^{4,5}$ In addition, there are differences in the pathogenesis of endoscopically positive and endoscopically negative reflux disease. This difference relates to different effectiveness of drugs that reduce the acidity of gastric contents when treating erosive and non-erosive GERD. ${ }^{6}$ As it turned out, in the case of a non-erosive form of reflux disease, the permeability of the esophageal mucosa is increased due to weakening of the integration of epithelial cells. The reason for this is a disruption of the tight junctions (TJ) of epithelial cells. ${ }^{?}$

A universal property of epithelial cells is to protect the internal environment of the body from damaging factors through the formation of the epithelial barrier. The surface of epithelial cells throughout the entire gastrointestinal tract is covered with a layer of mucus consisting mainly of mucins representing mainly glycoproteins. In the esophagus, the mucus produced by esophageal glands and goblet cells, together with bicarbonate, protein, ammonium and other saliva 
buffers, forms a pre-epithelial barrier on the way of aggressive reflux. The second line of defense are actually epithelial cells. The quality of their connection primarily depends on their tight junctions. The $\mathrm{TJ}$ are known to consist of several types of proteins as well as lipids providing barrier and transport functions of mucosa. Such proteins include claudins, occludins, and tricellulin, with claudins being the most important. ${ }^{8}$ Twenty four types of claudins were found in the human body; however, in the esophageal epithelium, claudins 1,2 , $3,4,5,7$ are of primary importance, and the number of claudins of different types changes in different diseases of the esophagus. ${ }^{9}$ E.g. in the case of GERD, a decrease in claudine 1 and 3 in the esophageal mucosa was found, while patients with biliary reflux had a decrease in the expression of claudin types 3 and $4 .{ }^{10}$ It is interesting to note that the level of claudin type 1 and 4 was higher in men compared with women with GERD. Moreover, the clinical manifestations of the disease in women with GERD were more pronounced and the quality of life was lower than in men. ${ }^{11}$

The basis for the treatment of GERD is to reduce the aggressiveness of the reflux agent and, first of all, to suppress gastric secretion by proton pump inhibitors (PPIs). The pathogenetically substantiated effect on the motility of the upper gastrointestinal tract and the tone of the lower esophageal sphincter has a lower effect on the symptoms and is used to potentiate the effect of antisecretory therapy in patients with a PPI-refractory GERD. ${ }^{12}$ A promising trend in the treatment of PPI resistant (especially non-erosive GERD) is an increase in the resistance of the esophageal mucosa to aggressive factors. In addition to the well-known cytoprotectors such as sucralfate and alginates, the use of a combination of hyaluronic acid and chondroitin sulfate has been proposed. ${ }^{13}$ A randomized study showed that such a combination potentiated the positive effect of PPIs in patients with non-erosive GERD, increasing the quality of life of these patients..$^{14}$ Given the above, we decided to assess the effect of the combination of sodium alginate and sodium hyaluronate on the course of GERD and to clarify the mechanisms of action of such a combination.

\section{Aim}

The aim of our study was to evaluate the clinical efficacy of esophageal mucus barrier correction by complex treatment with sodium hyaluronate and sodium alginate (Hyalera/Gastropiù Bustine)* application in patients with gastroesophagic reflux disease (GERD). Hyalera- registered product in Ukraine, Gastropiù Bustineregistered product in Italy.

\section{Materials and study methods}

We conducted a multicenter, randomized, open, comparative study with parallel monitoring to assess the efficacy and safety of combination therapy in patients with GERD with the use of sodium hyaluronate and sodium alginate (Hyalera/Gastropiù Bustine). The work was carried out at clinical bases of the Internal Medicine Department of P.L. Shupik National Medical Academy of Postgraduate Education (NMAPE), Gastroenterology Department, Kharkov Medical Academy of Postgraduate Education. The study included 72 patients with GERD aged 18 to 65years. Women were $30(41,7 \%)$, men $42(58,3 \%)$. The inclusion criterion for the study was the presence of GERD symptoms (especially heartburn) confirmed by endoscopic and morphological examinations. The exclusion criterion was the age under 18 years old or over 65 years old, immunodeficiency as a cause of esophagitis, intake of medicinal products that could damage the esophageal mucosa (potassium chloride, cytostatics, NSAIDs), severe concomitant diseases, QT interval elongation on ECG, alcoholism, drug addiction, mental disorders making difficult to understand and/or to contact with the patient.

Before treatment and 30days after, all patients were evaluated for GERD symptoms, general clinical and basic biochemical parameters, endoscopy with an assessment of the degree of damage to the esophagus according to the Los Angeles classification and they had a biopsy from the mucosa of the lower third of the esophagus. A pathomorphological study of biopsy samples of the esophagus of patients was carried out at the Department of Pathological and Topographic Anatomy of P.L. Shupik NMAPE. Biopsies analyzed in blinded manner always by the same pathologist.

The obtained biopsy specimens were preserved in a $10 \%$ solution of neutral buffered formalin, processed by a standard method, and embedded in paraffin. Serial $4-\mu \mathrm{m}$ thick histological sections were made from paraffin blocks on a rotational microtome NM 325 (Thermo Shandon, England), which were then stained with hematoxylin and eosin, and the PAS reaction was performed. In all cases, to determine the state of tight junctions /connections between epithelial cells, we performed an immunohistochemical assay (IHA) with a protein of the Claudin 1 family. To do this, sections were placed on Super Frost Plus adhesive glasses (Menzel, Germany). For high temperature treatment of antigen epitopes, citrate buffer with pH6, EDTA buffer, pH8, were used. Rabbit polyclonal antibodies to Claudine 1 (Master Diagnostica, Spain) were used. An Ultra Vision Quanto HRP detection system and the Quanto DAB chromogen (Thermo Fisher Scientific (USA)) were used.

The degree of Claudine expression was assessed using 2 semiquantitative methods $[15,16,17]$, which indicated the number of cells reacted with the marker and allowed objectifying the obtained data (Table 1). Microscopic examination and photo-archiving of the preparations were carried out using Carl Zeiss Primo Star light-optical microscopes with an Axiocam105 color camera, Carl Zeiss AX10 camera (Germany) and an Axiovision data processing system with 10 , 20, 40x magnifications.

Table I Assessment of claudine expression according to G. Sheehan method (2007)

\begin{tabular}{|c|c|c|c|c|}
\hline Staining intensity & Staining intensity score/ localization in epithelium* & Number of positive cells & Positive cell score & Total score \\
\hline Mild & $1 /+1$ & Less than $25 \%$ & 1 & $0 * *-3-$ mild \\
\hline Moderate & $2 /+2$ & $25-50 \%$ & 2 & 4-6-moderate \\
\hline Intensive & $3 /+3$ & More than $51 \%$ & 3 & 7-9- intensive \\
\hline
\end{tabular}

* - localization in epithelium - in surface parts, in other parts; $* *-0$ - reaction is absent

Claudin expression by $\mathrm{H}$ score (Shibutani M, 20I4, Suren D 20I4)

0 - Expression is absent.

$\mathrm{I}$ - Expression is observed in less than I/3 of membrane surface.

2 - Expression is observed in $1 / 3$ to $2 / 3$ of membrane surface.

3 - Expression is observed in more than $2 / 3$ of membrane surface. 
Patients were divided into 2 groups. The first group included 36 people (16 women $-44.4 \%$ and 20 men $(55.6 \%)$ ), the average age was $45.36 \pm 3.21$ years. Twelve patients were diagnosed with an erosive form, and 24 patients had a non-erosive GERD. The second group included 14 women $(38.9 \%)$ and 22 men $(61.1 \%)$, the average age of 42.94 \pm 3.18 years. Here, an erosive form was detected in 11, and

Table 2 GERD manifestations in the studied groups (before/after treatment)

\begin{tabular}{llllll}
\hline \multirow{2}{*}{ Manifestations } & \multicolumn{2}{l}{$\begin{array}{l}\text { Group I (sodium hyaluronate and sodium } \\
\text { alginate) }\end{array}$} & \multicolumn{2}{l}{ Group 2 (control) } & p \\
\cline { 2 - 6 } & $\mathbf{N}=\mathbf{3 6}$ & $\%$ & $\mathbf{N}=\mathbf{3 6}$ & $\%$ & $>0.05$ \\
\hline Heartburn & $36 / 2$ & $100 / 5.6$ & $36 / 8$ & $100 / 22.2$ & $>0.05$ \\
including nocturnal & $9 / 0$ & $25.0 / 0$ & $7 / 2$ & $19.4 / 5.6$ & $>0.05$ \\
Dysphagia & $12 / 0$ & $33.3 / 0$ & $11 / 2$ & $30.6 / 5.6$ & $>1.0$ \\
Eructation & $18 / 7$ & $50 / 19.4$ & $19 / 6$ & $52.8 / 16.7$ & $>1.0$ \\
Daily heartburn & $9 / 0$ & $25.0 / 0$ & $8 / 0$ & $22.2 / 0$ & $>0.05$ \\
Weekly heartburn & $27 / 2$ & $75.0 / 5.6$ & $28 / 8$ & $77.8 / 22.2$ & $>0.05$ \\
Non-erosive & $24 / 2$ & $66.7 / 5.6$ & $25 / 9$ & $69.4 / 25.0$ & $>0.1$ \\
A-B by LA & $10 / 0$ & $27.8 / 0$ & $11 / 1$ & $30.5 / 2.8$ & $>1.0$ \\
C-D by LA & $2 / 0$ & $5.5 / 0$ & $1 / 0$ & $2.8 / 0$ & \\
\hline
\end{tabular}

$p$ - differences between groups

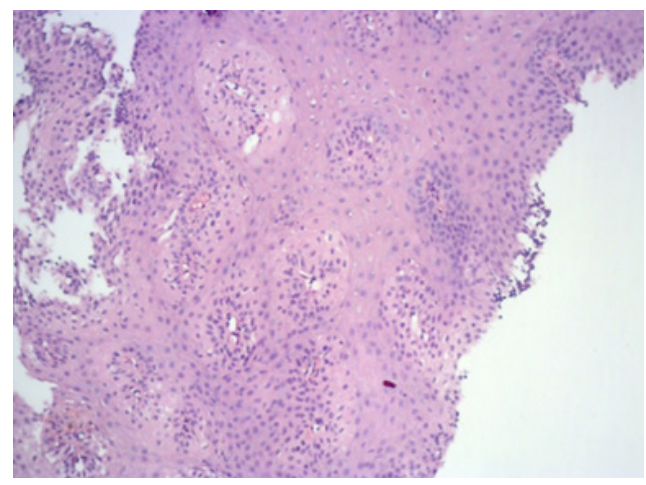

Figure I A fragment of the esophageal mucosa with severe degenerative changes, papillomatosis, focal proliferation of the epithelium. Hematoxylin and eosin staining, 50x magnification.

A PAS reaction showed a heterogeneous focal decrease in color in areas of inflammation, proliferation and degeneration of the epithelium, which reflected a decrease in mucus secretion, a sharp decrease in mucus secretion in the submucosal layer (Figure 2).

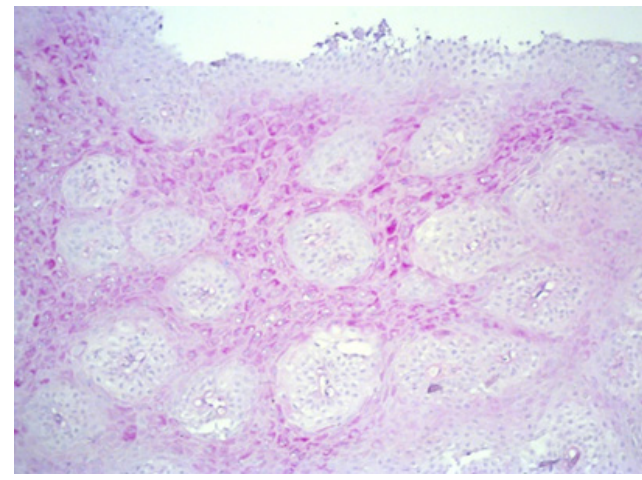

Figure 2 A fragment of esophageal mucosa, an area of papillomatosis, a sharp decrease in mucus formation. PAS reaction, 50x magnification.

\section{(a)}

non-erosive in 25 patients. As can be seen from Table 2, no significant differences in the manifestations of the disease were observed between the groups. The morphological study in the group of patients before treatment showed pronounced degenerative changes, erosion areas, parakeratosis, irregular regenerative changes, zones of papillomatosis and focal proliferation (Figure 1). 


\section{Results and discussion}

After a 30 day course, improvement was achieved in both groups of patients. In the first group, a significant clinical improvement was observed after sodium hyaluronate and sodium alginate intake. Heartburn disappeared in $94.4 \%$ of patients, the symptoms of heartburn persisted in $5.6 \%$ of patients but its frequency was only 1-2 times a week. Patients had no episodes of nocturnal heartburn and dysphagia. On average, the number of heartburn episodes decreased from $4.97 \pm 0.36$ per week to $0.31 \pm 0.15(p<0.001)$. The number of patients with eructation decreased by $60 \%$. An endoscopic examination did not detect erosions in patients, only $5.6 \%$ of patients had mild hyperemia in the lower third of the esophagus.

Interesting data were obtained during a morphological study of biopsy samples of the esophageal mucosa. After 30days of combination therapy, a significant decrease in the degree of inflammation in the epithelium and areas of epithelial degeneration was observed, although the zones of its regeneration remained. Patients who received sodium hyaluronate and sodium alginate in the complex had slight signs of degenerative changes in the form of dystrophy of varying degrees, papillomatosis cells, manifestations of regenerative changes in their esophagus (Figure 4). A PAS reaction showed a significant increase in the synthesis of mucus and an increase in the thickness of its layer on the epithelium surface. Some patients of this group had normalization of mucus secretion in the submucosal regions (Figure 5).

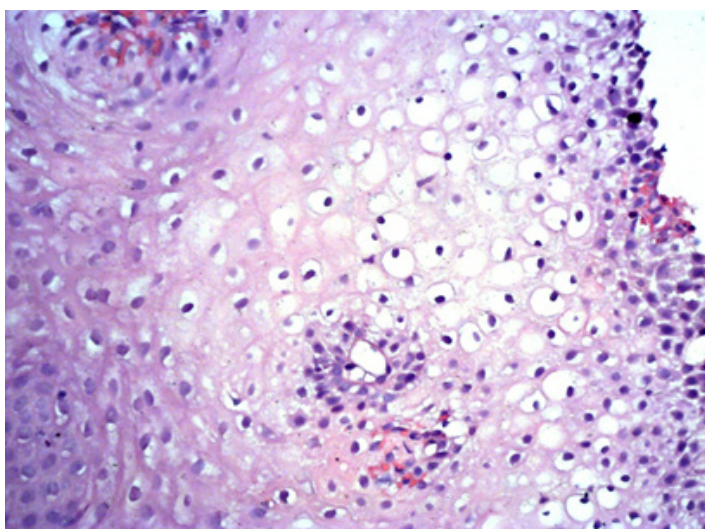

Figure $4 \mathrm{~A}$ fragment of esophageal mucosa with degenerative changes, signs of regeneration. Hematoxylin and eosin stain, 200x magnification.



Figure 5 A fragment of esophageal mucosa, an area of papillomatosis, a decrease in the reaction in the areas of epithelial proliferation. PAS reaction, 50x magnification.
An immunohistochemical assay with claudine 1 in patients of this group after sodium hyaluronate and sodium alginate (Hyalera/ Gastropiù Bustine) intake predominantly showed an increase in the degree of staining from moderate to intensive (score 2 or 3 ). Positive membrane expression of claudine was observed in more than 51\% (score 3) epithelial cells. The total expression score ranged from 6 to $9, \mathrm{H}$-score expression was predominantly 3 - (more than in $2 / 3$ of the surface of the epithelial cell membranes) (Figure 6).

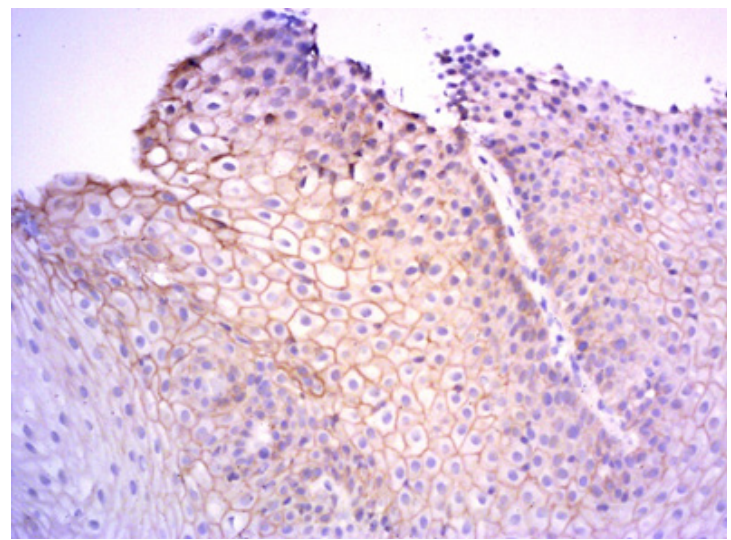

Figure 6 Fragment of the esophagus, pronounced and widespread (up to $80 \%$ of the epithelial cell membranes) expression of the Claudine I protein of tight junctions. IHC with Claudin I, I00x magnification.

The second (control) group of patients had less significant positive changes. Heartburn persisted in almost a quarter of patients, and $5.6 \%$ of them had heartburn at night. The frequency of occurrence of heartburn ranged from 1 to 5 times a week. On average, the frequency of heartburn decreased from $4.64 \pm 0.32$ to $0.92 \pm 0.24$ times a week $(\mathrm{p}<0.001)$. Moreover, dysphagia persisted in $5.6 \%$ of patients, and eructation disturbed $16.7 \%$ of patients. During the follow up endoscopic examination, signs of esophagitis were found in $25.0 \%$ of patients (including $5.6 \%$ of patients without complaints on heartburn). Grade A erosive esophagitis according to the Los Angeles classification after treatment persisted in one patient $(2.7 \%)$.

Morphologically, the patients of this group had a decrease in the severity of inflammation. At the same time, mucus synthesis increased moderately, and claudine 1 expression remained virtually unchanged and remained low. It should be noted that the frequency of heartburn in the first group after the therapy was almost three times lower than in the reference group $(\mathrm{p}=0.035)$ and the patients did not have nocturnal heartburn. Moreover, dysphagia was absent in the first group (it persisted in almost $6 \%$ in the second group), while the incidence of eructation after the therapy was identical in both groups (19.4 and 16.7\%). Endoscopically, the patients of the second group had esophagitis 4.5 times more frequently than in the first group and it was erosive in one patient. It is also necessary to emphasize the high safety of sodium hyaluronate and sodium alginate (Hyalera/Gastropiù Bustine) intake. No clinically significant side effects of the therapy were observed in both groups (both new symptoms as well as clinical and biochemical findings).

How can these results be explained? A decrease in the frequency of heartburn and the absence of its nocturnal episodes due to the additional intake of sodium hyaluronate and sodium alginate (Hyalera/ Gastropiù Bustine) may be due to the well-known property of alginate to form a protective film on the surface of the gastric contents, due to which, when reflux occurs (especially in a horizontal position), 
it is alginate (mechanical barrier) and not aggressive acid, which penetrates into the esophageal lumen. At the same time, alginate sodium is effective not only in the case of acidic but also biliary reflux, and is also characterized by high safety and therefore can be used not only in adults but even in children. ${ }^{13,18}$ Another equally important effect of sodium hyaluronate and sodium alginate (Hyalera/Gastropiù Bustine) is esophagoprotection. According to results of experimental and clinical studies, sodium alginate and hyaluronate can increase the resistance of esophageal mucosa to damaging effects of reflux agents. ${ }^{13,19}$ As our study showed, such esophagoprotection is associated with an increase in claudine 1 expression on the surface of esophageal mucosa cytoplasm. This results in a decrease in the permeability of the epithelium due to the strengthening of tight intercellular junctions. It is equally important for esophagoprotection that we have found an increase in the synthesis of protective mucus, which forms a barrier on the epithelial surface that prevents damage to epithelial cells. It should also be noted that similar positive effects of sodium hyaluronate and sodium alginate (Hyalera/Gastropiù Bustine) were noted not only in the esophagus, but also in the stomach, although this is a topic for a different study. Administration of sodium hyaluronate and sodium alginate (Hyalera/Gastropiù Bustine) demonstrated high safety.

Thus, mucus barrier correction in patients with GERD could be a perspective target of the treatment. In GERD patients the addition of a proton pump inhibitor, prokinetic, sodium hyaluronate and sodium alginate (Hyalera/Gastropiù Bustine) to standard therapy can significantly (compared with the control group) reduce the frequency and severity of heartburn, the degree of damage to the esophagus according to esophagoscopy, eliminate dysphagia and nocturnal heartburn. At the same time, modification of esophageal mucus barrier correlated with clinical improvement. Morphological changes in the esophageal mucosa, with decrease in the degree of inflammation and degeneration of the epithelium, an increase in the synthesis of protective mucus and the integration of the epithelium due to claudine 1 in tight junctions induce a clinical symptoms normalisation. Consequently, sodium hyaluronate and sodium alginate stimulates the preepithelial and epithelial barriers of the esophagus, which is especially important in patients with a non-erosive form of GERD that is resistant to conventional antisecretory therapy.

\section{Conflicts of interest}

All authors declared there are no conflicts of interest.

\section{Acknowledgements}

None.

\section{Funding}

None.

\section{References}

1. Richter JE, Rubenstein JH. Presentation and Epidemiology of Gastroesophageal Reflux Disease. Gastroenterology. 2018;154(2):267276
2. Eusebi LH, Ratnakumaran R, Yuan Y, et al. Global prevalence of, and risk factors for, gastro-oesophageal reflux symptoms: a meta-analysis. Gut. 2018;67(3):430-440.

3. Tkach S.M. Modern possibilities of increasing the effectiveness of treatment of gastroesophageal reflux disease. Gastroenterol. 2014;52(2):82-88.

4. Dorofeyev AE, Afanasiev MV, Rassohina OA, et al. Some mechanisms of esophagoprotection in patients with gastroesophageal reflux disease and their correction. Suchasna Gastroenterol. 2011;57(1):78-83.

5. Tack J, Pandolfino JE. Pathophysiology of Gastroesophageal Reflux Disease. Gastroenterology. 2018;154(2):277-288.

6. Gyawali CP, Azagury DE, Chan WW, et al. Nonerosive reflux disease: clinical concepts. Ann N Y Acad Sci. 2018 Dec;1434(1):290-303.

7. Woodland P, Sifrim D. Esophageal mucosal integrity in nonerosive reflux disease. J Clin Gastroenterol. 2014;48(1):6-12.

8. France MM, Turner JR. The mucosal barrier at a glance. $J$ Cell Sci. 2017;130(2):307-314.

9. Oshima T, Miwa H. Gastrointestinal mucosal barrier function and diseases. J Gastroenterol. 2016;51(8):768-778.

10. Björkman EV, EdeboA, Oltean M, et al. Esophageal barrier function and tight junction expression in healthy subjects and patients with gastroesophageal reflux disease: functionality of esophageal mucosa exposed to bile salt and trypsin in vitro. Scand J Gastroenterol. 2013;48(10):1118-2116.

11. Kim JJ, Kim N, Park JH, et al. Comparison of Tight Junction ProteinRelated Gene mRNA Expression Levels between Male and Female Gastroesophageal Reflux Disease Patients. Gut Liver. 2018;12(4):411-419.

12. Mermelstein J, Chait Mermelstein A, Chait MM. Proton pump inhibitorrefractory gastroesophageal reflux disease: challenges and solutions. Clin Exp Gastroenterol. 2018;11:119-134.

13. Savarino E, Zentilin P, Marabotto E, et al. Drugs for improving esophageal mucosa defense: where are we now and where are we going? Ann Gastroenterol. 2017;30(6):585-591.

14. Savarino V, Pace F, Scarpignato C, et al. Randomised clinical trial: mucosal protection combined with acid suppression in the treatment of non-erosive reflux disease - efficacy of Esoxx, a hyaluronic acid-chondroitin sulphate based bioadhesive formulation. Aliment Pharmacol Ther. 2017;45(5):631642 .

15. Shibutani M, Noda E, Maede K, et al. Low expression of claudin-I and presence of poorly-differentiated tumor cluster correlation with poor prognosis in colorectal cancer. Anticancer Res. 2013;33(8):3301-3306.

16. Sheehan GM, Kallakury BV, Sheehan CE, et al. Loss of claudin-1 and -7 and expression of claudin-3 and -4 correlate with prognostic variables in prostatic adenocarcinomas. Hum Pathol. 2007;38(4):564-569.

17. Suren D, Yildirim M, Kaya v, et al. Loss of tight junction proteins (Claudin 1,4 and 7) correlates with aggressive behavior in colorectal carcinoma. Med Sci Monit. 2014;20:1255-1262.

18. Salvatore S, Ripepi A, Huysentruyt K, et al. The Effect of Alginate in Gastroesophageal Reflux in Infants. Paediatr Drugs. 2018;20(6):575-583.

19. Yurtçu M, Arbag H, Toy H, et al. The healing effects of tissue glues and healing agent locally applied on esophageal anastomoses. Int $J$ Pediatr Otorhinolaryngol. 2010;74(1):43-44. 